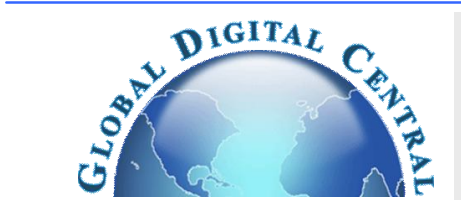

Frontiers in Heat and Mass Transfer

\title{
TRANSIENT RAYLEIGH-BÉNARD-MARANGONI CONVECTION ENHANCED GAS-LIQUID SOLUTE TRANSFER IN THIN LAYERS
}

\author{
Muthasim Fahmy ${ }^{\dagger}$, Zhifa Sun \\ Department of Physics, University of Otago, Dunedin, 9016, New Zealand
}

\begin{abstract}
A gas-liquid solute transfer process initiated in a closed vessel can exhibit Rayleigh-Bénard-Marangoni (RBM) convection enhanced mass transfer. For short exposure times experimental and theoretical results demonstrate that for deep liquid systems prior to solute penetration across the depth of the fluid, the stability thresholds of the system decreases with time. For thin liquid layers at longer exposure times the mass transfer enhancement under RBM convection can be affected in two ways: (1) solute penetration to the bottom liquid-solid boundary causing a departure from a penetration type concentration profile; (2) solute penetration to the top gas-solid boundary in the gas phase resulting in deviations of mass transfer Biot number from the penetration type of Biot number. These two effects have been investigated by imposing non-diffusing boundary conditions in the liquid phase as well as the gas phase. For short contact times, the critical thresholds of convection evaluated via a quasi-static stability analysis under non-diffusing boundary conditions are consistent with those under penetration type concentration profile. However, at longer exposure times when solute has completely penetrated the entire liquid depth, there can only be a limited period of time when convective instability is possible. Within this period there is a local maximum of convective intensity, thereby opening up the possibility of optimising gas-liquid mass transfer operations with respect to Rayleigh and Marangoni convection. Experimental results supporting these predictions are presented.
\end{abstract}

Keywords: Rayleigh-Bénard-Marangoni convection, transient enhanced heat and mass transfer, Boundary effects.

\section{INTRODUCTION}

Heat- and mass-transfer enhancement due to Rayleigh-Bénard (RB) convection and Bénard-Marangoni (BM) convection are well known phenomena and continue to be of importance in many engineering applications such as evaporators, absorbers, heat exchangers and falling film reactors (Rayleigh, 1916; Pearson 1959; Buffone and Sefiane, 2004; Scheid et al., 2008). Theoretical and experimental analyses of RB and/or BM convection enhanced mass transfer therefore have practical significance. Analysis of systems which simultaneously exhibit the Rayleigh effect and the Marangoni effect are referred to as RayleighBénard-Marangoni (RBM) problems (Nield, 1964; Lebon and Cloot, 1982).

In applications such as gas-liquid absorption or desorption, convective instabilities are strongly influenced by a time varying nonlinear concentration profile in the case of a stationary solute transfer systems or a spatially varying non-linear concentration profile in the case of a parallel gas-liquid flow solute transfer. The stability of such systems have been extensively studied (e.g., Kim et al., 2007; Pearson, 1959; Scriven and Sternling, 1964; Sun and Fahmy, 2006; Lu et al., 1997). Most of these studies have focused on various stability analyses of systems with penetration type concentration profile to determine critical onset time, when the system first becomes unstable. In these systems the penetration regime can be maintained over a large period of time after initial contact. In the case of parallel flow solute transfer systems, this allows one to study the critical behaviour on the basis of the gas-liquid interface velocity, since under penetration condition one can reasonably neglect the variation of fluid velocity across the liquid layer depth (Byers and King, 1967). While the treatment of fluid flow is simplified by a penetration type solute transfer regime, their stability analysis is complicated in that the time rate of change of solute concentration gradients can be comparable or larger than the growth rate of disturbances (Gresho and Sani, 1971). In this situation the classic quasi-static analysis (Lick, 1965) based on the frozen time model may not be valid. Other approaches to stability, such as amplification theory and propagation theory are less satisfactory in that they retain subjective decisions on initial conditions of disturbances or criteria for critical onset (Gresho and Sani, 1971). However, in gasliquid solute transfer system with thin liquid layers, the quasi-static analysis is known to predict critical thresholds that are in satisfactory agreement with experiment if the effect of surface viscosity in the Gibbs adsorption layer is considered (Sun, 2006). In thin layer parallel flow gas-liquid solute transfer systems, a quasi-static analysis together with the simplification of flow velocity treatment under penetration conditions show that the critical Rayleigh and Marangoni numbers will continue to monotonically decrease with time (Sun and Fahmy, 2006).

Analysis of RBM convection in parallel flow solute transfer systems beyond the penetration region in thin liquid layers is complicated by the fact that the velocity of the liquid layer cannot be assumed equal to the surface velocity and the non-linear concentration profile is influenced by the non-linear velocity profile (Bird, et al., 2007). However, this difficulty can be avoided by studying the RBM convection in a stationary gas-liquid system, in which case the base velocity has a constant zero value. A straightforward quasi-static analysis in a stationary gas-liquid system is therefore a feasible option to study the effect of solute penetration to the bottom liquid-solid. Furthermore it is relatively easier to make accurate measurements of solute transfer rates in a stationary solute transfer system, for instance by monitoring the pressure response to an applied pressure step. This 
method of studying gas-liquid mass transfer, originally due to Plevan and Quinn (1966) will be used in this study to experimentally test the transient behaviour of gas-liquid mass transfer in a stationary liquid in thin layers. To our knowledge, the time dependent behaviour of critical parameters at long times when the solute has penetrated to the bottom boundary and the behaviour of mass transfer enhancement under these conditions have not been previously studied. In this paper we study the time dependent RBM convection enhanced mass transfer behaviour of unsteady gas-liquid mass transfer in thin liquid layer systems via a quasi-static analysis. In particular we seek to determine how the critical Rayleigh and Marangoni numbers point vary with time as solute penetrates to the bottom boundary and how this affects the mass transfer rates at long times by considering a gas-liquid solute transfer system which is initially stationary and then subject to step change in concentration driving force thereby initiating a transient mass transfer process that eventually becomes unstable with respect to Rayleigh or Marangoni convection. We will show how this can lead to only a limited time interval where RBM convection is possible.

\section{MODEL DESCRIPTION}

\subsection{Unperturbed Concentration Profiles}

The solute transfer process between a stationary horizontal gas-phase is illustrated schematically in Fig. 1.

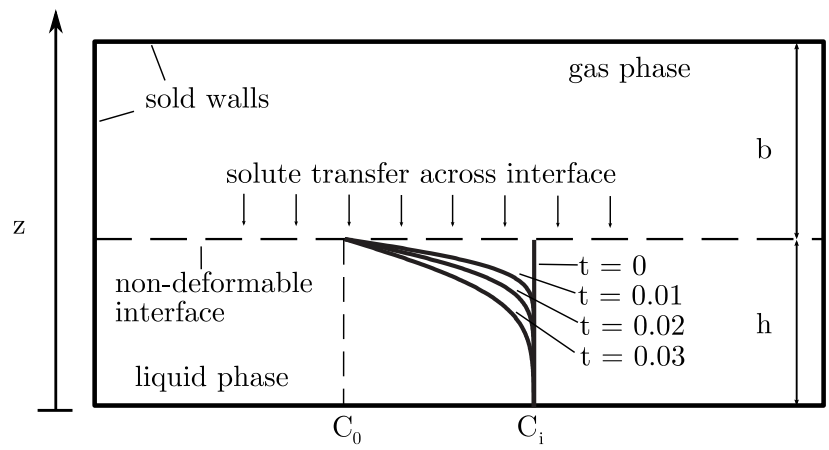

Fig. 1 Physical Model ( $\mathrm{t}$ is the non-dimensionalised time)

A thin liquid layer of thickness $h$, with an initial solute concentration of $C_{0}$, is brought into contact with a gas layer of thickness $b$ which consists of a mixture of the solute and saturated vapour of the liquid species. The concentration of solute in each of the two phases is assumed to be uniform prior to contact. In such a situation, the solute transport across the interface for the unperturbed states of the system is then governed by (Bird et al., 2007).

$\partial_{t} C(t, z)=D_{l} \partial_{z z} C(t, z)$

with initial and boundary conditions given by,

$$
\begin{aligned}
& C(t=0, z)=C_{0} \\
& C(t, z=h)=C_{i} \\
& \partial_{z} C(t, z=0)=0
\end{aligned}
$$

The time dependent concentration profile is obtained from solution to the system (1)-(4) as (Debnath, 2005),

$$
\frac{C(t, z)-C_{0}}{C_{i}-C_{0}}=\sum_{n=0}^{\infty}(-1)^{n}\left[\operatorname{erfc}\left(\frac{(2 n+1) h-z}{\sqrt{4 D_{l} t}}\right)+\operatorname{erfc}\left(\frac{(2 n+1) h+z}{\sqrt{4 D_{l} t}}\right)\right]
$$

This is in contrast to the penetration concentration profile (Bird et al., 2007),

$$
\frac{C(z)-C_{i}}{C_{0}-C_{i}}=\operatorname{erf}\left[\frac{h-z}{\sqrt{4 D_{l} t}}\right]
$$

obtained for short contact times, when the liquid layer can be considered infinitely deep.

\subsection{Governing Equations for the Perturbed States}

The governing equations describing the time evolution of the velocity and concentration fields for the stationary system influenced by the Rayleigh effect and/or Marangoni effect can be obtained via the continuity equation, momentum balance equation, and solute mass balance in the bulk phase and the interface. If we define the velocity fields $\mathbf{v}^{\prime}$ as the sum of base state velocity field $\mathbf{V}=(U, V, W)$ and a small perturbation velocity field $\mathbf{v}=(u, v, w)$, we have,

$\mathbf{v}^{\prime}=\mathbf{V}+\mathbf{v}$

and similarly introduce the concentration field as the sum of the base concentration and the perturbation concentration field,

$C^{\prime}=C+\theta$

then, after introducing the non-dimensionalising scales $h$ for distance, $h^{2} / D_{l}$ for time and $\Delta C=C_{0}-C_{i}$ for concentration, the linearised and non-dimensionalised governing equations for the perturbation fields in the liquid bulk phase $0<z<1$, can be written as follows (here we only reproduce final model equations and we refer the reader to Sun and Fahmy (2006) for further details):

$\operatorname{Ra} \nabla_{1}^{2} \theta+\nabla^{4} w=0$

$\partial_{t} \theta-\nabla^{2} \theta+w \partial_{z} C=0$

$\nabla^{2}\left[\nabla_{1}^{2} v+\partial_{y z} w\right]=0$

$\nabla^{2}\left[\nabla_{1}^{2} u+\partial_{x z} w\right]=0$

The boundary conditions at the gas liquid interface at non-dimensional coordinate $z=1$ are

$w=0$

$\partial_{z} \theta-\theta \mathrm{Bi}=0$

$\partial_{z z} w-\operatorname{Ma} \nabla_{1}^{2} \theta=0$

and at the bottom liquid-solid boundary at non-dimensional coordinate $z=0$ we have,

$\partial_{z} \theta=0$

$u=v=w=0$

$\partial_{z} w=0$

It is noted that for brevity, the same symbols for the dimensional variables, e.g. concentration, time, and coordinates, have been used for the corresponding non-dimensionalised variables in Eqs. (9)-(18) and the dimensionalised variables in Eqs. (1) to (6) and the equations below.

Since the main objective of this paper is to investigate the effect of the non-diffusing boundaries on the critical parameters and the mass 
transfer enhancement behaviour of RBM convection, Gibbs adsorption effects have been neglected in the present analysis (Brian, 1971; Brian and Ross, 1972; Palmer and Berg, 1972; Sun and Fahmy, 2006).

The non-dimensional groups in the above equations are defined as follows:

Rayleigh number:

$\mathrm{Ra}=\frac{g \alpha_{0} h^{3}\left(C_{b}-C_{s}\right)}{v_{l} D_{l}}$

Marangoni number:

$\mathrm{Ma}=\frac{\sigma_{0} h\left(C_{b}-C_{s}\right)}{\mu_{l} D_{l}}$

Biot number:

$\mathrm{Bi}=\frac{H k_{c g} h}{R T D_{l}}$

In Eqs. (19) and (20), $C_{b}$ is the concentration of solute at the liquidsolid boundary (non-dimensional coordinate $z=0$ ) and $C_{s}$ is the solute concentration at the gas-liquid interface (non-dimensional coordinate $z=1$ )

\subsection{Quasi-static Analysis and Numerical Methods}

Since the numerical solutions of the quasi-static models are straightforward and involve no subjective decisions on the initial conditions of perturbations and on the criterion for the onset time (or position of the onset), the quasi-static analysis technique has been used in this paper to determine the behaviour of critical onset of RBM convection where solute has penetrated to the non-diffusing solid bottom boundary (Sparrow et al., 1964; Vidal and Acrivos, 1966; Brian et al., 1967; Currie, 1967; Gresho and Sani, 1971; Davis and Choi, 1977). Critical points of the Rayleigh and Marangoni instabilities obtained using the quasi-static analysis technique has been compared with the experimental results in absorption and desorption of carbon dioxide into and from shallow layers $(<3 \mathrm{~mm})$ of two organic solvents: methanol and toluene in a gas-liquid contactor with a contact length of $11 \mathrm{~cm}$ by Sun (2006). Using reasonably estimated values for the surface viscosity and the surface dilational viscosity of the Gibbs adsorption layers, the calculated values of the overall-system critical concentration are in satisfactory agreement with the measured data (Sun, 2006). In the stationary gas-liquid system where convective patterns are not limited to rolls as is the case in parallel flow systems (Davis and Choi, 1977; Sun and Fahmy, 2006), one would seek normal mode solutions of the form,

$(u, v, w, \theta)=[\hat{U}(z), \hat{V}(z), \hat{W}(z), \hat{\Theta}(z)] \exp \left[i\left(k_{x} x+k_{y} y\right)\right]$

where $k_{x}$ and $k_{y}$ are the components of a horizontal plane wave vector, $\mathbf{k}=\left(k_{x}, k_{y}\right)$, on an $\mathrm{x}$-y plane orthogonal to the z-direction and $i$ is the unit imaginary number. Using this relation for the perturbed variables, the principle of exchange of stability has been adopted implicitly (Chandrasekhar, 1981; Davis and Choi, 1977; Gresho and Sani, 1971). The normal mode equations that completely describe the critical onset can be written succinctly in terms of the plane wave number $k=\sqrt{k_{x}^{2}+k_{y}^{2}}$ as given in Eq. (23), where the operator $D=d / d z$.

$$
\left[\begin{array}{cccccccc}
\left(D^{2}-k^{2}\right)^{2} & -\mathrm{Ra} k^{2} & 0 & 0 & 0 & 0 & 0 & 0 \\
-\partial_{z} C & \left(D^{2}-k^{2}\right) & 0 & 0 & 0 & 0 & 0 & 0 \\
0 & 0 & 1 & 0 & 0 & 0 & 0 & 0 \\
0 & 0 & 0 & D & 0 & 0 & 0 & 0 \\
0 & 0 & 0 & 0 & 1 & 0 & 0 & 0 \\
0 & 0 & 0 & 0 & 0 & 1 & 0 & 0 \\
0 & 0 & 0 & 0 & 0 & 0 & 0 & (D+B i) \\
0 & 0 & 0 & 0 & 0 & 0 & D^{2} & k^{2} \mathrm{Ma}
\end{array}\right]\left[\begin{array}{c}
\hat{W} \\
\hat{\Theta} \\
\hat{W}_{z=0} \\
\hat{W}_{z=0} \\
\hat{\Theta}_{z=0} \\
\hat{W}_{z=1} \\
\hat{W}_{z=1} \\
\hat{\Theta}_{z=1}
\end{array}\right]=\mathbf{0}(23)
$$

If the gas layer can be considered infinitely deep, then with a penetration model of mass transfer, the gas phase mass transfer coefficient is given by $k_{c g}=\sqrt{D_{g} / \pi t}$. Here $D_{g}$ is the diffusivity of solute in the gas phase and $t$ is the dimensioned time. Then, from Eq. (21) we can obtain the mass transfer Biot number under penetration conditions as given in Eq. (24).

$\mathrm{Bi}_{p}=\frac{1}{\sqrt{\pi}}\left(\frac{H}{R T}\right)\left(\frac{D_{g}}{D_{l}}\right)^{\frac{1}{2}}\left(\frac{h^{2}}{D_{l} t}\right)^{\frac{1}{2}}$

In a real experiment involving gas-liquid solute transfer, the assumption of infinite depth for the gas phase is unlikely to be valid for long times since the diffusivity of solute in the gas phase is significantly larger than the diffusivity of solute in the liquid phase. Under these conditions the solute would penetrate to the top solid boundary in gas phase significantly faster than the solute penetration to the bottom boundary in the liquid phase. Once the solute has penetrated the entire depth of the gas phase, Eq. (24) would no longer describe the mass transfer Biot number. Therefore, to investigate the effects of solute penetration to the bottom liquid-solid boundary, it is also necessary to consider effects of solute penetration to the top gas-solid boundary on the Biot number. To obtain the appropriate expression for gas phase mass transfer coefficient in a finite depth gas phase, one must solve a diffusion problem analogous to Eqs. (1) through (4), for the gas phase to yield the gas phase concentration distribution, from which one can determined the gas phase mass transfer coefficient. Carrying out this procedure, we have obtained the gas phase mass transfer coefficient as (see Appendix A for additional detail),

$k_{c g}=\left(\frac{D_{g}}{\pi t}\right)^{\frac{1}{2}} \phi_{g}$

where the factor $\phi_{g}$ describing the deviation from penetration type mass transfer conditions is given by,

$\phi_{g}=\frac{\sum_{n=0}^{\infty}(-1)^{n}\left\{\exp \left[-n^{2} Z_{n}\right]-\exp \left[-(n+1)^{2} Z_{n}\right]\right\}}{1-\sum_{n=0}^{\infty} 2(-1)^{n} \operatorname{erfc}\left[\left(n+\frac{1}{2}\right) \sqrt{Z_{n}}\right]}$

and where

$Z_{n}=\left(\frac{D_{l}}{D_{g}}\right)\left(\frac{b}{h}\right)^{2}\left(\frac{h^{2}}{D_{l} t}\right)$

The Biot number for both short and long exposure times, $\mathrm{Bi}_{f}$, is obtained by substituting for the gas phase mass transfer coefficient from Eq. (25) into the definition Eq. (21). Thus we have, 


$$
\mathrm{Bi}_{f}=\frac{H h}{R T D_{l}}\left(\frac{D_{g}}{\pi t}\right)^{\frac{1}{2}} \phi_{g}
$$

Introducing the non-dimensionalising scales $h$ for coordinates and $h^{2} / D_{l}$ for time, Eq. (28) can be written as,

$\mathrm{Bi}_{f}=\left[\frac{1}{\sqrt{\pi}}\left(\frac{H}{R T}\right)\left(\frac{D_{g}}{D_{l}}\right)^{\frac{1}{2}}\left(\frac{h^{2}}{D_{l} t}\right)^{\frac{1}{2}}\right] \phi_{g}$

where the term in square brackets can be identified as the Biot number in the penetration regime, $\mathrm{Bi}_{p}$, given by Eq.(24). Thus, the Biot number for both short and long exposure times is related to the Biot number in the penetration regime by,

$\mathrm{Bi}_{f}=\mathrm{Bi}_{p} \phi_{g}$

We solve the eigenvalue problem defined by Eqs. (23) using a Chebyshev spectral method (Trefethen, 2000; Weideman and Reddy, 2000) to obtain the critical Rayleigh and Marangoni numbers. Namely the system on $0 \leq z \leq 1$ shown in Fig. 2 is linearly scaled to $-1 \leq z \leq 1$ and the solutions $W(z)$ and $\Theta(z)$ are approximated on $N$ Chebyshev collocation points given by,

$z_{j}=\cos \left[\frac{(j-1) \pi}{N-1}\right], \quad j=1, \cdots, N$

and represented by the discretised interpolant function,

$$
f(z)=\sum_{j=1}^{N} \frac{(-1)^{n} f\left(z_{j}\right)}{c_{j}\left(z-z_{j}\right)} / \sum_{j=1}^{N} \frac{(-1)^{n}}{c_{j}\left(z-z_{j}\right)}
$$

where

$c_{j}= \begin{cases}2 & j=1 \text { or } N \\ 1 & \text { otherwise }\end{cases}$

The operator $D$ defined by $d f(z) / d z=D f(z)$ can then be approximated by an $N \times N$ matrix as described in Trefethen (2000) and Weideman (2000), with higher order operators given by $D^{(n)}=\left(D^{(1)}\right)^{n}$. The differential eigenvalue problem in Eqs. (23) is thereby transformed into a matrix eigenvalue problem which can be readily solved. The grid size $N$ was incremented until the calculated eigenvalues converged to a solution and further increasing $N$ leaves the solution unchanged. The implementation of the Chebyshev spectral method was validated against the method of variational principles reported in Sun and Fahmy (2006), under penetration concentration profile conditions. The spectral method has the advantage in that it is easy to implement and that an ad hoc selection of basis functions with special analytical properties is not required (Feit et al., 1982).

\section{THEORETICAL RESULTS}

\subsection{Effect of Mass Transfer Biot Number}

Figure 2 contrasts the penetration concentration profile $(\mathrm{PEN})$ with non-diffusing bottom boundary (NDBB) profile of Eq. (5). In Fig. 2, for the purpose of illustration the dimensionless concentration $C_{i}$ is assumed to be equal to unity and the dimensionless bulk concentration $C_{0}$ is assumed to be zero at the beginning of the process. For short exposure times under penetration concentration profile conditions in the liquid phase, which corresponds to the dimensionless times less than approximately 0.03 (Sun and Fahmy, 2006), the concentration of solute at the bottom liquid-solid boundary is $C_{b}=C_{0}$. For $t>0.03$, according to the penetration concentration profile, $C_{b}$ is greater than $C_{0}$ and the vertical concentration gradient is no longer zero at the bottom liquidsolid boundary as would be required by the existence of the solid nondiffusing bottom boundary, and therefore penetration model for mass diffusion no longer applies and the concentration profile of Eq. (5) must be used instead. In the limiting case of large $t$, the NDBB profile is seen to approach the equilibrium condition where the solute concentration is uniform across the depth of the liquid layer. The penetration profile, on the other hand, approaches a constant gradient profile. This is a physically invalid situation since this predicts a sustained solute flux across the liquid layer for an indefinite period of time from a constant interface concentration. Since we have a nondiffusing rigid boundary at the bottom of the liquid phase, the solute accumulation must necessarily oppose further addition of solute and evolve towards an equilibrium state with no further solute flux into the liquid.

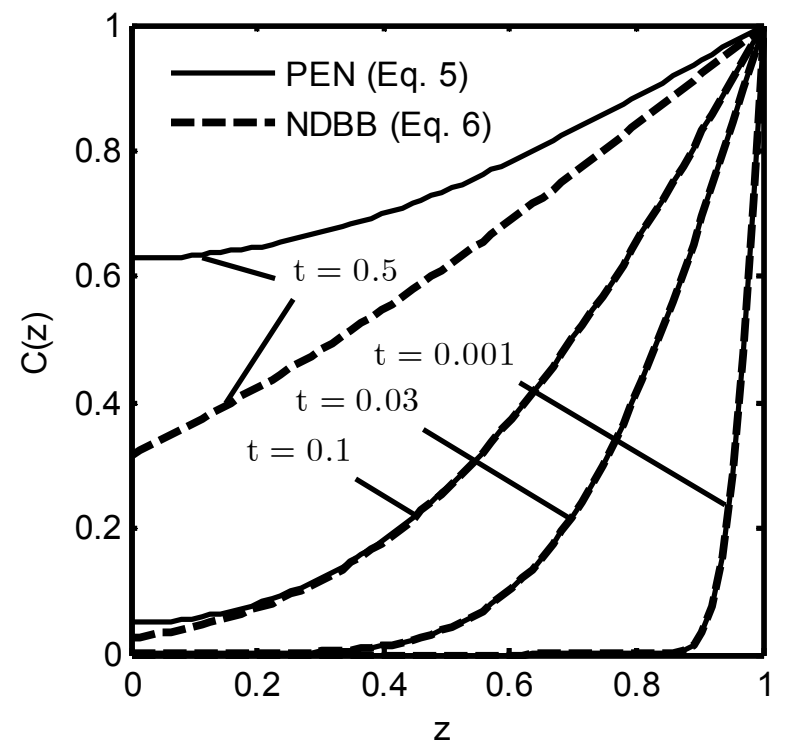

Fig. 2 Comparison between penetration and non-diffusing bottom boundary concentration profiles ( $\mathrm{z}$ and $\mathrm{t}$ are dimensionless)

In Fig. 3 we consider the effect of finite gas phase thickness on mass transfer Biot number. The solid lines represent the variation of Biot number in a finite thickness gas phase under a non-diffusing top boundary condition as calculated from Eq. (30) at a constant gas-liquid diffusivity ratio $D_{g} / D_{l}=5000$. In Fig. 3 the circle, square and triangle points on the solid lines represent the marginal point between a perfectly diffusing top boundary and a non-diffusing top boundary in the gas phase corresponding to gas-liquid thickness ratios $b / h=10$, 15 and 25 respectively. These points correspond to a non-dimensional time of approximately $0.03(b / h)^{2}\left(D_{l} / D_{g}\right)$. As can be expected the departure from penetration conditions happens sooner for small values of gas-liquid thickness ratio $b / h$. The dashed line represent the Biot number as obtained from the penetration model of mass transfer (Eq. (24)) in the gas phase. Since, for the values of $b / h$ presented in Fig 3, we have $(b / h)^{2}<D_{g} / D_{l}$, the effects of solute penetration to the top 
solid boundary of the gas phase become pronounced well before the liquid phase marginal time of approximately 0.03 .

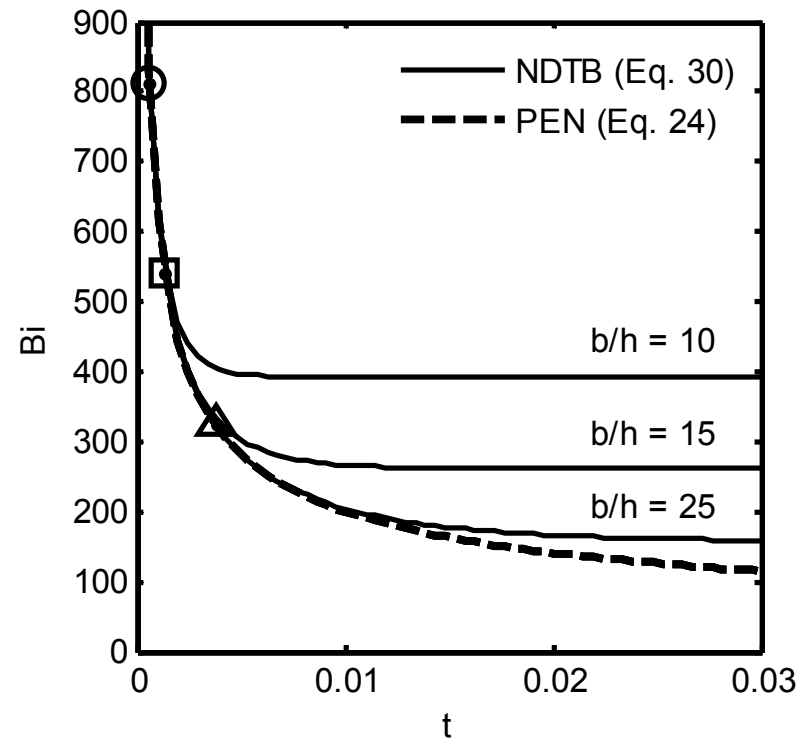

Fig. 3 Variation of mass transfer Biot number with gas-liquid thickness ratio and dimensionless time.

Figure 4. shows the effect of the diffusivity ratio on the finite thickness gas phase Biot number at a constant gas-liquid thickness ratio of $b / h=20$. In Fig. 4 the circle, square and triangle points on the solid line represents the marginal point where the penetration model of the mass transfer for the gas phase correspond to gas-liquid diffusivity ratios $D_{g} / D_{l}=6000,5000$ and 4000 respectively.

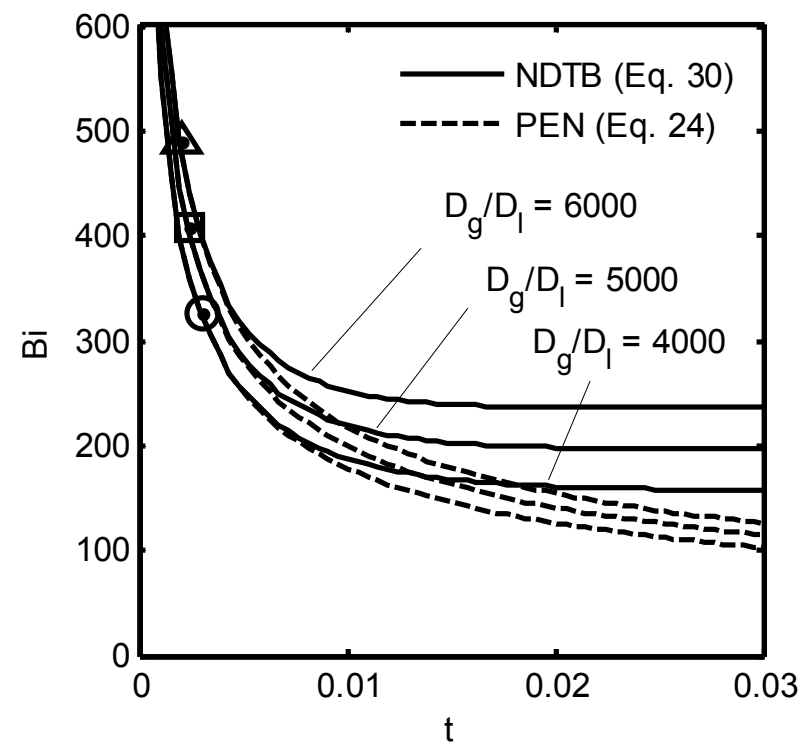

Fig. 4 Variation of mass transfer Biot number with gas-liquid diffusivity ratio and dimensionless time.

The dashed line represents the Biot numbers evaluated under penetration conditions in gas phase. Figures 3 and 4, taken together show that effect of a finite gas phase on the Biot number is that after a non-dimensional time of approximately $0.03(b / h)^{2}\left(D_{l} / D_{g}\right)$, the Biot number decreases more slowly than would be the case under penetration mass transfer conditions, at a rate determined by the gasliquid thickness ratio and the gas-liquid diffusivity ratio.
Considering solute penetration in the liquid phase, it can be seen from Fig 2, that the solute concentration at the bottom liquid-solid boundary is time dependent. If the gas phase can be considered to have an infinite thickness, then from Eq. (5), the normalized concentration difference between the bottom boundary and the interface is given by,

$$
\frac{C_{b}(t)-C_{i}}{C_{0}-C_{i}}=\left[1-\sum_{n=0}^{\infty} 2(-1)^{n} \operatorname{erfc}\left(\frac{(2 n+1) h}{\sqrt{4 D_{l} t}}\right)\right]
$$

which rapidly decreases when the non-dimensionalised time is greater than approximately 0.03 . If the gas thickness is finite, the absorption or desorption process will give rise to a variation in pressure with time. This in turn will result in a variation of interface concentration $C_{s}(t)$ with time. In systems where the solute transfer is liquid phase controlled, the variation in system pressure (and hence the variation in interface concentration) can be estimated by employing a mass balance at the interface, based on the liquid phase mass transfer coefficient. For a sufficiently large gas thickness, one finds that variation in interface concentration is small so that the variation in system driving force $C_{b}-C_{s}$ is dominated by the variation in $C_{b}$ as given by Eq. (34). Consequently, the absolute values of the system operation Rayleigh and Marangoni numbers decrease with increasing contact time. As is evident from Eqs. (19) and (20) we see that Rayleigh and Marangoni numbers are proportional to $C_{b}-C_{s}$ and the signs of the Rayleigh and Marangoni numbers depend on whether the fluid physical properties $\alpha_{0}$ and $\sigma_{0}$ are positive or negative, and whether the solute transfer is an absorption or desorption process. For the purpose of illustration, we have chosen operating parameters such that the proportionality constant is positive in Eqs. (19) and (20). The bottom boundary and interface concentrations are non-dimensionalised by taking $C_{0}-C_{i}$ as a unit of concentration and the diffusivity of the liquid phase has been taken as a typical value of $D_{l}=10^{-9} \mathrm{~m}^{2} \mathrm{~s}^{-1}$. With these parameters a unit of dimensionless time corresponds to approximately 1 hour in a typical physical system with a typical organic liquid absorbing or desorbing a solute such as carbon dioxide with a liquid thickness of the order of a few millimetres.

\subsection{Transient Bénard-Marangoni Problem}

To compare the present theory against predictions based on the penetration model, we first look at the special case where $\mathrm{Ra}=0$, the Bénard-Marangoni (BM) problem (as would the case for solute transfer in microgravity conditions). We consider a test system for which $D_{g} / D_{l}=5000$ and $H / R T=0.5$. In addition we consider the solute transfer process to be a desorption process with test conditions $C_{s}=0$, $C_{0}=10, \quad \sigma_{0}=10^{-6} \mathrm{Nm}^{2} \mathrm{~mol}^{-1}, \quad \mu_{l}=5 \times 10^{-4} \mathrm{~N} \mathrm{~s} \mathrm{~m}^{-2} \quad$ and $h=1 \times 10^{-3} \mathrm{~m}$. In all of the following analysis, it is assumed that after the critical point, BM and RB effects do not significantly change the concentration profile predicted by Eq. (5). The instantaneous system operation Marangoni number can then be evaluated from Eqs. (20) and (34). The time evolutions of the critical Marangoni number and the system operation Marangoni number are illustrated in Fig. 5. The dashed lines in Fig. 5 have been evaluated for three different values of gas-liquid thickness ratio, as indicated in the figure.

In the initial penetration regime up to the marginal point (dimensionless time $t \approx 0.03$ represented by the black circle point) between a perfectly diffusing wall $\Theta(z=0)=0$ and a non-diffusing wall $D \Theta(z=0)=0$ for the liquid phase, the solute has not penetrated to the bottom. As would be expected within this penetration region the predicted critical Marangoni number based on the penetration boundary condition and non-diffusing bottom boundary condition are almost the 
same. However, shortly after the solute has penetrated to the bottom boundary, the decrease in the critical Ma slows down and reaches a minimum value and thereafter increases with increasing time, eventually exceeding the system operation Marangoni number.

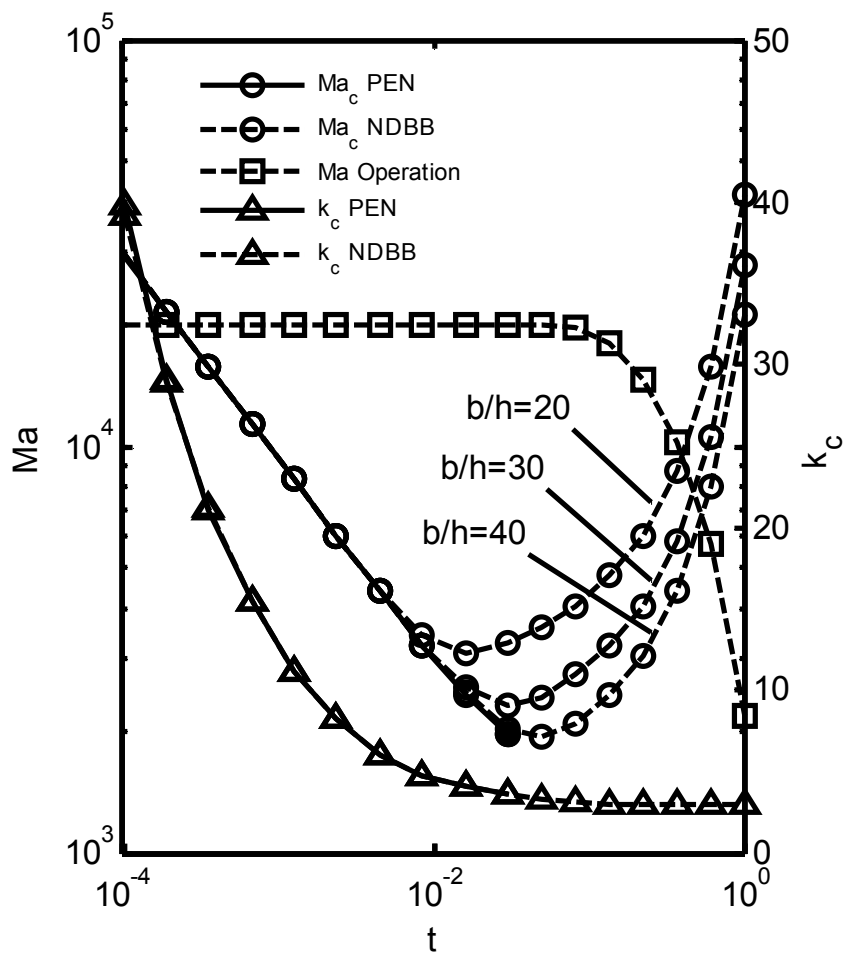

Fig. 5. Variation of critical $\mathrm{Ma}, k$, and the system operation $\mathrm{Ma}$ with dimensionless contact time and gas-liquid thickness ratio for the BM problem.

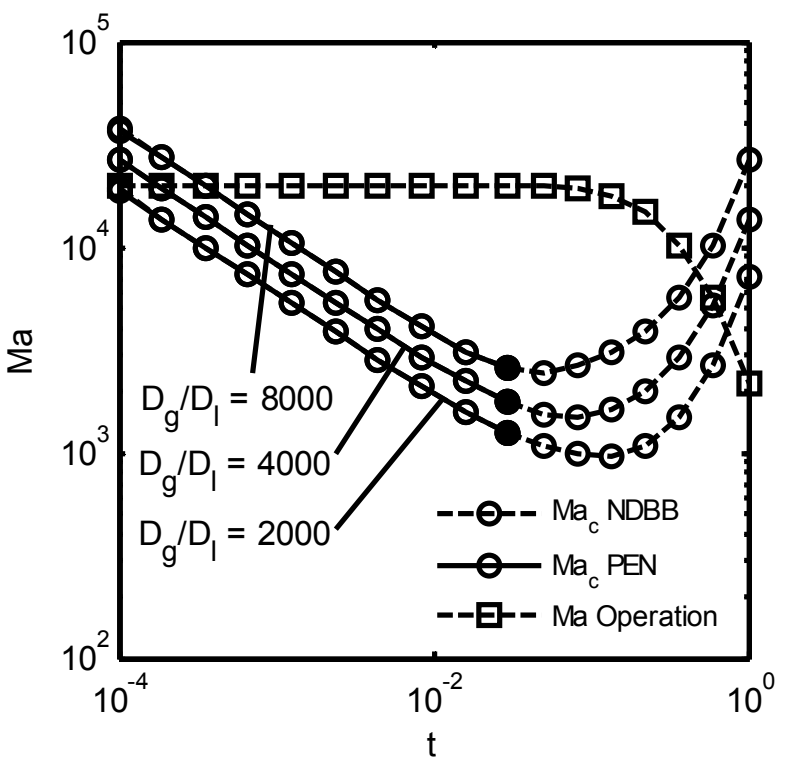

Fig. 6. Variation of critical and system operation Ma, with dimensionless contact time and gas-liquid diffusivity ratio for the BM problem.

Immediately after initial gas-liquid contact the Marangoni number has a very large initial value (orders of magnitude larger than the system operation Marangoni number). In this region the system is stable with respect to the Marangoni effect. Once the critical Ma falls below the system operation Ma, Marangoni convection begins. Immediately after critical onset, the system over criticality parameter
$\left(\mathrm{Ma}-\mathrm{Ma}_{c}\right) / \mathrm{Ma}_{c}$ increases approximately linearly with time. Some time after critical contact, the over criticality parameter is further reduced since the system operation $\mathrm{Ma}$ is no longer constant but varies as $M a \propto\left[C_{b}(t)-C_{s}(t)\right]$. We therefore get a local maximum of $\left(\mathrm{Ma}-\mathrm{Ma}_{c}\right) / \mathrm{Ma}_{c}$ where Marangoni convection is expected to be most intense, and when mass transfer rate is a maximum. With further increase in time, the system approaches its phase equilibrium conditions with the system operation Ma approaching zero. Thus a consequence of solute penetration to the non-diffusing boundaries is that we get a single restricted time interval when Marangoni convection occurs and mass transfer is enhanced. For long contact times, the convective motion can initiate and then dissipate within a certain time interval and the width of the interval increases with increasing driving force. The convective time interval is strongly dependent on gas-liquid thickness ratio, with the interval decreasing with thickness ratio. The width of the convective time interval is also strongly dependent on the gas-liquid diffusivity ratio as show in Fig. 6. Large gas-liquid diffusivity ratios results in a longer convective time interval. Finite thickness of the gas phase and liquid phase was found to have negligible effect on the critical wavenumbers $k_{c}$. That is the size of the convective cells is not sensitive to solute penetration to the solid boundaries in either the gas phase or the liquid phase.

\subsection{Transient Rayleigh-Bénard Problem}

A behaviour similar to the case of the pure BM convection was observed with pure Rayleigh convection where $\mathrm{Ma}=0$. This is illustrated in Fig. 7. In this instance the system-operation Rayleigh number varies as $\operatorname{Ra} \propto\left[C_{b}(t)-C_{s}(t)\right]$, and once again this gives rise to a single restricted time interval where Rayleigh convection occurs and mass transfer is enhanced.

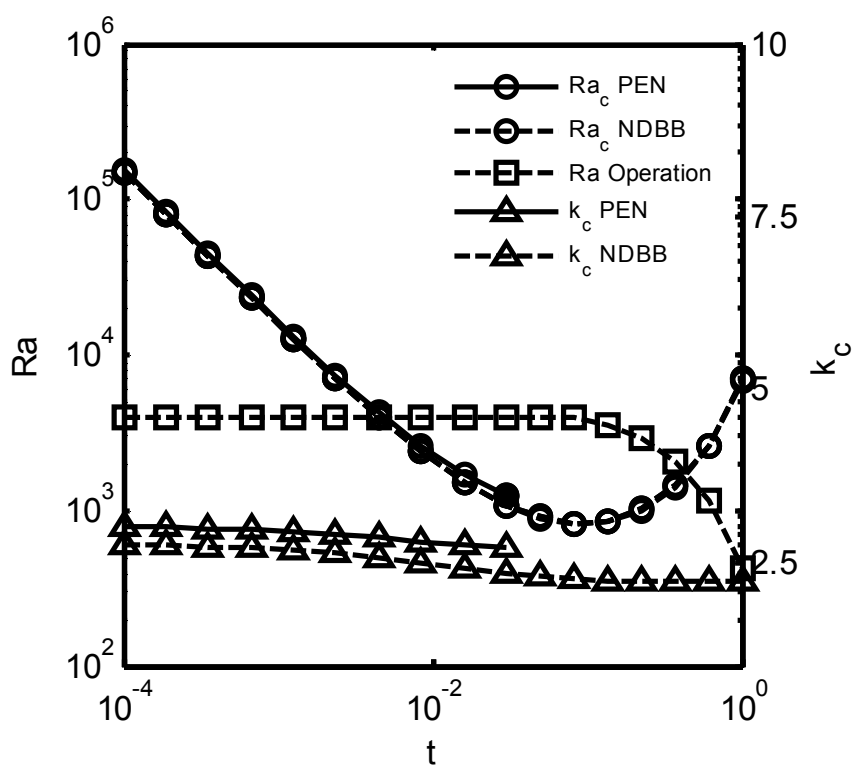

Fig. 7. Variation of critical Ra, critical $k$, and the system operation $\mathrm{Ra}$ with dimensionless contact time for the $\mathrm{BM}$ problem $(\mathrm{Ma}=0)$.

In Fig. 7, the test conditions are $H / R T=0.5, C_{0}=0, C_{s}=1$, $\alpha_{0}=-10^{-6} \mathrm{Nm}^{2} \mathrm{~mol}^{-1}, \mu_{l}=5 \times 10^{-4} \mathrm{~N} \mathrm{~s} \mathrm{~m}^{-2}$ and $h=2 \times 10^{-3} \mathrm{~m}$. Since the variations in $D_{g} / D_{l}$ and $b / h$ have very little effect on the critical Rayleigh number of the RB problem, in Fig. 5, we only show the critical curve using constant values $D_{g} / D_{l}=5000$, and $b / h=30$. 
A comparison of the critical wavenumber evaluated under penetration and non-diffusing boundary conditions indicate that the presence of a non-diffusing solid boundary at the bottom of liquid layer would result in a slightly increased size of convective cells in the pure $\mathrm{RB}$ problem.

\subsection{Transient Rayleigh-Bénard-Marangoni Problem}

Figure 8 compares the concentration fluctuations at the marginal point $t \approx 0.03$ using the penetration boundary conditions and the nondiffusing boundary condition for the BM problem. For the plots in Fig. 8 , the test conditions are $H / R T=0.5, C_{0}=1, C_{s}=0$, $\sigma_{0}=10^{-6} \mathrm{Nm}^{2} \mathrm{~mol}^{-1}, \quad \mu_{l}=5 \times 10^{-4} \mathrm{~N} \mathrm{~s} \mathrm{~m}^{-2}$ and $h=2 \times 10^{-3} \mathrm{~m}$, $D_{g} / D_{l}=5000, b / h=30$ and $\mathrm{Ra}=0$.

As expected the penetration boundary condition enforces zero fluctuations at $z=0$ with $\Theta=0$ and $\partial_{z} \Theta \neq 0$ and the NDBB model enforces $\partial_{z} \Theta=0$ with $\Theta \neq 0$. The result is that the predicted concentration fluctuation differs significantly between the two models as $z$ approaches zero, with the penetration model underestimating the fluctuation near $z=0$. A similar comparison of the velocity fluctuation (not shown in this paper) gave no significant difference between the velocity fluctuations computed using the two models for entire domain $0 \leq z \leq 1$. Nevertheless the difference in the concentration fluctuations can be expected to have some effect on the convective mass transfer enhancement predicted from the two models, at least close to a critical point.

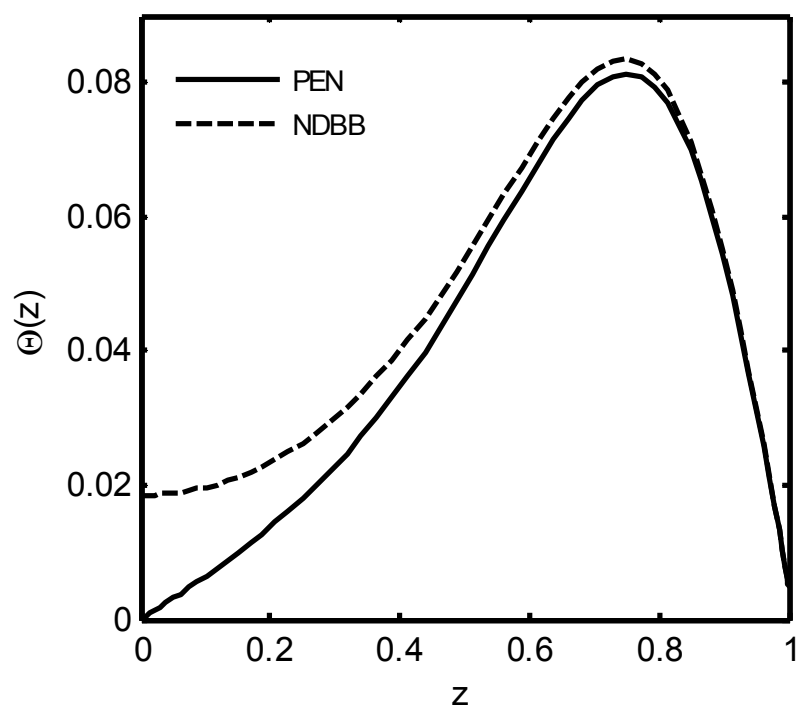

Fig. 8. Variation of concentration perturbations with dimensionless $\mathrm{z}$, ( $\mathrm{Ra}=0$, dimensionless $t=0.03$ ).

From weakly non-linear analyses, it is known that in the slightly overcritical region the mass transfer Sherwood number increases linearly (Sun and Yu, 2006) with the quantity $\int_{0}^{1} \Theta(z) W(z) d z$, which is the area bounded between the curve of $\Theta(z) W(z)$ and the $\Theta(z) W(z)=0$ line shown in Fig. 9. Since there is contribution to mass transfer enhancement from across the entire depth of the liquid, this suggests that the true mass transfer enhancement due to convection would be underestimated by penetration theory as dimensionless time $t$ approaches 0.03 from zero. For systems that become critical at larger times, Fig. 9 (which has been evaluated under non-diffusing boundary conditions) indicates that the convective mass transfer intensity would decrease with the critical time as solute penetrates to the bottom boundary.

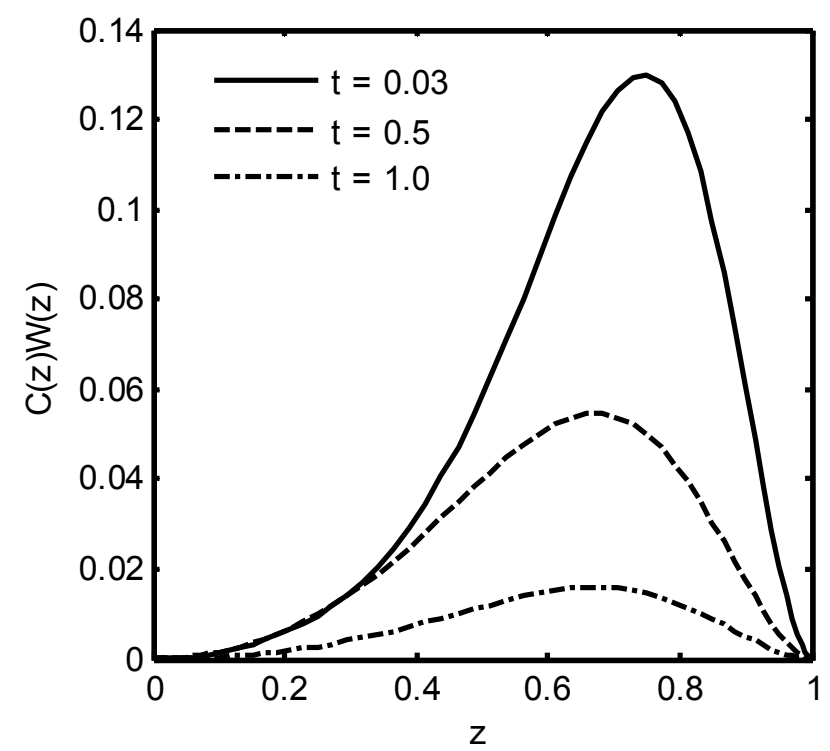

Fig. 9. Variation of the product of concentration and velocity perturbations with dimensionless $\mathrm{z} \quad(\mathrm{Ra}=0$ and $\mathrm{t}$ is dimensionless).

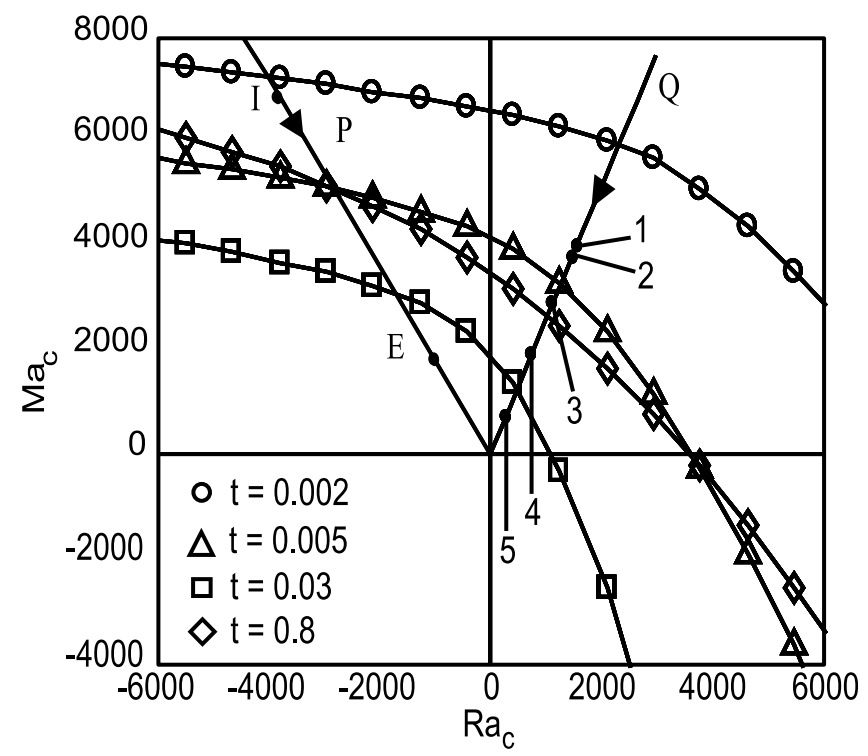

Fig. 10. Variation of critical $\mathrm{Ma}$ and $\mathrm{Ra}$ with contact time for the RBM problem ( $\mathrm{t}$ is dimensionless).

In Fig. 10 we look at the effect of the non-diffusing bottom boundary for the more general case where both Rayleigh and Marangoni convection occur simultaneously. In a real physical process, even though the system operation $\mathrm{Ra}$ and $\mathrm{Ma}$ both decrease with contact time (similar to that shown in Fig. 6 and Fig. 7), the ratio $\mathrm{Ma} / \mathrm{Ra}$ is fixed by the fluid physical properties and by the process: absorption or desorption. Thus a straight line, e.g. line P in Fig. 10, on which $\mathrm{Ma}=$ constant $\times \mathrm{Ra}$, depicts the system operation line on the Ma-Ra plane. The system follows along the arrow direction on the operation line from an initial contact time represented by I to the final point represented by E. Depending on the values of $\alpha$ and $\sigma$ and $\Delta C$, RBM convection can only occur in either the first, second or fourth 
quadrants. It can also be noted that similar to the behaviour for the cases of the BM problem and the RB problem we have the possibility that the critical threshold first decreases and then increases in the general RBM problem. Along the operation line Q shown in Fig. 10, the points $1,2,3,4$, and 5 correspond to non-dimensional contact times $t=0.03,0.1,0.23,0.45$ and 0.8 , respectively.

Under typical conditions with a solute such as carbon dioxide and an organic liquid such as methanol with a thickness of a few millimetres, these non-dimensional times correspond to contacting dimensioned times of approximately $2,5,15,30$, and 50 minutes. The critical Ra-Ma curves at $t=0.002,0.005,0.03$ and 0.8 correspond to approximately $0.1,0.5,2$ and 50 minutes from initial contact respectively. These critical curves show that the critical values of Ma and $\mathrm{Ra}$ decrease with contact time and then increase, similar to the behaviour shown in Figs 5, 6 and 7. For time less than about 2 minutes, the system operating point remains approximately fixed at point 1 , taking approximately 3 minutes to go from point 1 to point 2 , while the critical parameters $\mathrm{Ra}$ and $\mathrm{Ma}$, starting from very large values, rapidly approach and then fall below the system operation $\mathrm{Ra}$ and Ma values. Thus the curves shown in Fig. 10 indicate that system instability will start within 0.5 minutes of contact. In the remaining time of the contact process, the system operation $\mathrm{Ra}$ and $\mathrm{Ma}$ approach the equilibrium value of zero. By the time the system reaches point 5 on the operation line corresponding to approximately 50 minutes, the values of the system operation Ra and Ma have fallen below the critical values and Rayleigh and Marangoni convection subsides. Therefore, it is the competition between these two effects, the variation of the critical threshold and the variation of the system operation $\mathrm{Ra}$ and Ma that will determine the bounds of the time interval when convective instability can occur. This behaviour is the main difference between the present study and the results reported by Sun and Fahmy (2006), where only short contact times were investigated.

\section{EXPERIMENTS}

\subsection{Experimental Setup and Procedure}

The prediction of restricted time intervals for convective instability in gas-liquid contactors for long contact times has yet to be confirmed experimentally. Nevertheless the collapse of mass transfer enhancement factor, which was defined by Brian and Ross (1972) as the ratio of experimentally measured mass transfer coefficient to that predicted by penetration model of mass transfer, after a long exposure time has been observed in wetted-wall columns (Hozawa et al., 1984). In particular the mass transfer coefficient measurements by Hozawa et. al. (1984) in the methanol- $\mathrm{CO}_{2}$ system are consistent with a local maximum of mass transfer enhancement. While strictly speaking, the flow system would require special treatment of base state velocity profile variations (Bird et al., 2007) one would expect similar behaviour between the flow system and stationary system.

The collapsing of mass transfer enhancement has been observed in our laboratory with mass transfer processes in stationary gas-liquid systems. Here we report some initial measurements of mass transfer enhancement factors in a stationary liquid associated with the physical absorption of carbon dioxide using an apparatus similar to that of Plevan and Quinn (1966) in their study of the effect of monomolecular films on gas absorption into quiescent liquids and Blair and Quinn (1969) in their study of cellular convection. The apparatus used is illustrated schematically in Fig. 11.

The apparatus consists of a cylinder of $99.98 \%$ purity instrument grade carbon dioxide (1) which is saturated with the solvent vapour in the saturator (2). The mixture of saturated vapour and carbon dioxide is then introduced into a pressure vessel (3) by keeping valve V1 open and valves V2 and V5 closed until a predetermined pressure in the approximate range $100 \mathrm{kPa}$ to $200 \mathrm{kPa}$, read out from pressure gauge (4) is reached. The items (6) and (10) are two identical cylindrical cells made of aluminium and closed at both ends with $1 \mathrm{~cm}$ thick BK7 optical glass. The glass windows and the aluminium cylinder seal against Teflon $\mathrm{O}$ rings. Each cell has an internal diameter of $4.984 \pm 0.002 \mathrm{~cm}$. The height of each cell measured from glass window to glass window is $6.016 \pm 0.002 \mathrm{~cm}$. The cell (6) serves as the absorption test cell into which the liquid sample is introduced by removing the top glass window.

After introducing a small amount of degassed liquid into the test cell, both cells are evacuated using the vacuum pump (12) by opening V6, V7 and keeping all other valves closed to remove air that may have been introduced during the liquid transfer. After degassing the vacuum is disconnected by closing V7 and both cells are slowly brought to a
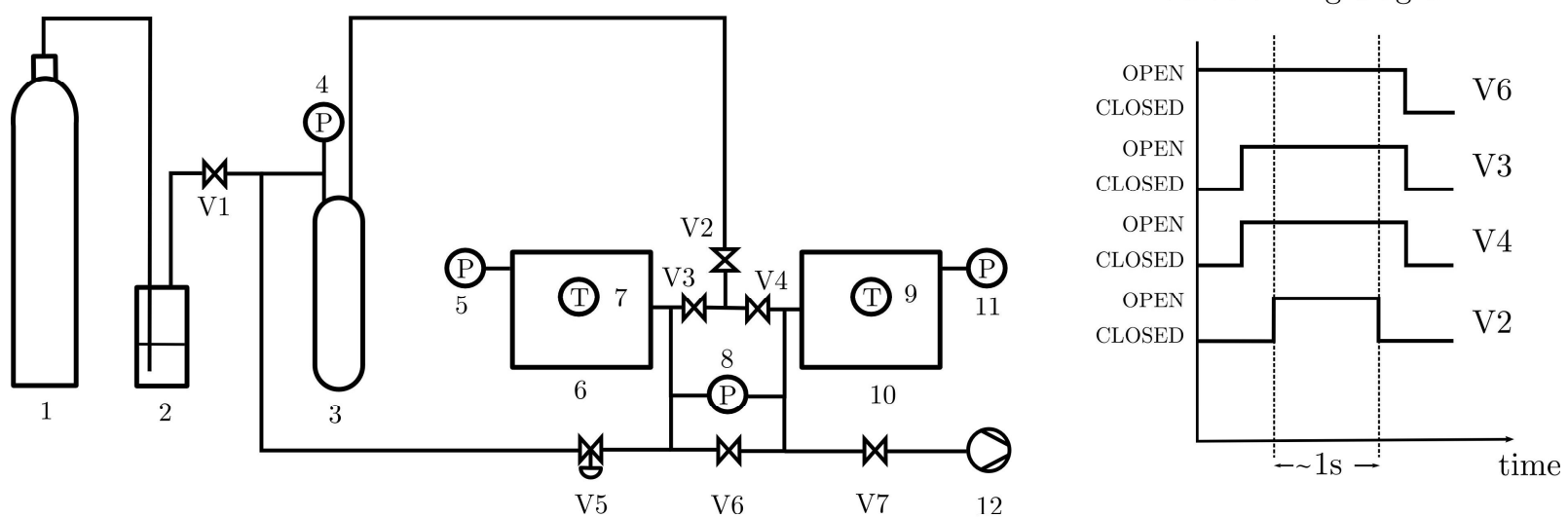
1. CO2 cylinder
5, 11. Absolute pressure transmitter
2. Saturator
6. Test cell
3. Pressure vessel
7, 9. Thermocouples
4. Pressure gauge
8. Differential pressure transmitter

V1, V5, V7. Manually operated valves

V2, V3, V4, V6. Pneumatically actuated valves

12. Vacuum pump

Fig. 11. Schematic of the apparatus used for mass transfer measurement 
predetermined initial pressure $P_{0}$ by introducing gas from the pressure vessel via control valve V5. The system is then allowed to equilibrate for approximately 12 hours at a constant room temperature. Observing a steady pressure at the end of this equilibrating process serves as a leak test for the system.

Valves V2, V3, V4 and V6 are high speed, pneumatically actuated and can be triggered electronically in the sequence shown in Fig. 11. As can be seen from the Fig. 11 this sequence simultaneously and momentarily connects both the test cell and the reference cell to the pressure vessel thereby applying a pressure step starting with an initial equilibrium pressure $P_{0}$ and terminating at a final pressure $P_{i}$. The entire pressure stepping operation takes approximately three seconds. The applied pressure step shifts the equilibrium of the gas-liquid system and initiates an absorption process. The ensuing absorption of the gaseous solute into the liquid phase in the confined cell results in a pressure decay from which the solute transfer rate can be determined

\subsection{Mass Transfer Rates and Enhancement Factors}

The analysis of the pressure decay to measure mass transfer rates in a gas-liquid system essentially consists of an initial-boundary value problem. Specifically we consider the one dimensional diffusion of solute in the quiescent liquid contained within the test cell such that the liquid bottom boundary is located at the vertical coordinate $z=0$ and the gas-liquid interface at $z=h$. Diffusion within the liquid can then be described by Eq. (1). We suppose that the equilibrium concentration of solute in the liquid before the pressure step is applied is $C_{0}$, so that initial condition is $C(z, 0)=C_{0}$. We further assume that an instantaneous equilibrium exists between the gas phase and the liquid interface at all times. Then, immediately before the pressure step, the partial pressure of $\mathrm{CO}_{2}$ in the gas phase is related to the concentration of $\mathrm{CO}_{2}$ at the interface via Henry's law as $P_{0}=H C_{0}$, and immediately after the pressure step we have $P_{i}=H C_{i}$. That is, at the gas liquid interface we have a time varying boundary condition $C(t, h)=P_{g}(t) / H$. The instantaneous solute balance at the gas-liquid interface then requires that (Plevan and Quinn, 1966; Farajzadeh et al., 2007)

$$
\left(\frac{d C}{d t}\right)_{z=h}=-\frac{Z A R T D_{l}}{V H}\left(\frac{\partial C}{\partial z}\right)_{z=h}
$$

where $Z$ is the compressibility of the gas phase, $A$ is the interface area, $R$ is the molar gas constant, $T$ is the system temperature, and $V$ is the gas volume. Eq. (35) and the following equations pertaining to experimental analysis have been given in dimensioned variable. Plevan and Quinn (1966) obtained an expression for the pressure response by assuming that the liquid phase was semi infinite. Since we wish to study the behaviour of the system when the solute has penetrated to the bottom, it would be prudent to obtain an equivalent expression for the pressure response for the finite-depth liquid, namely with a boundary condition $\partial_{z} C(t, 0)=0$. The time dependent boundary condition however complicates the solution to the problem. Sheika et al. (2005) in their estimates of diffusivity of gases in Bitumen using the pressure decay method obtained an expression for the finite-depth model in the Laplace transformed domain and used numerical Laplace transform inversion to obtain the pressure response. We however seek a more convenient analytic solution similar to that of Plevan and Quinn as a means of better defining of the initial contact time $t=0$ which is important in accurately determining short critical contact times for RBM convection. The accurate determination of short critical contact times can be extremely difficult in transient gas-liquid solute transfer due to difficulties in precisely defining the initial contact time in any given experiment. In order to obtain an analytical solution to the expected pressure response we follow the approach of Zhang et al. (2000) where they assume that once the initial interface concentration is specified, the concentration in the liquid phase and hence the concentration gradient at the interface at all future times is closely approximated by the solution to the diffusion equation with the appropriate boundary conditions, which in the present case is given by (5). The pressure response is then assumed to be dictated by the requirement of instantaneous solute balance at the interface as given by (27). This approach of determining the pressure response using an equilibrium boundary condition has been compared with more sophisticated quasi-equilibrium and non-equilibrium boundary conditions by Tharanivasan et al. (2004) where they conclude that the relative agreement between prediction and experiment for the various boundary conditions depends on the particular solute-solvent system. According to Etminan et. al., (2010), this indicates that the physics of time varying interface mass transfer is not well understood. Using the equilibrium boundary condition, we obtained the following pressure response which corresponds to Eq. (6) of Plevan and Quinn for the infinite-depth liquid.

$$
\frac{P_{g}(t)-P_{i}}{P_{0}-P_{i}}=\frac{Z A R T}{V H} \sqrt{D_{l} t} \frac{2}{\sqrt{\pi}} \sum_{n=0}^{\infty}(-1)^{n}\left[X_{n}-Y_{n}\right]
$$

where

$$
\begin{aligned}
& X_{n}=\exp \left(-\frac{h^{2}(n+1)^{2}}{D_{l} t}\right)+\exp \left(-\frac{h^{2} n^{2}}{D_{l} t}\right) \\
& Y_{n}=\sqrt{\frac{\pi h^{2}}{D_{l} t}}\left[(n+1) \operatorname{erf}\left(\frac{h(n+1)^{2}}{\sqrt{D_{l} t}}\right)-n \operatorname{erf}\left(\frac{h n^{2}}{\sqrt{D_{l} t}}\right)-1\right]
\end{aligned}
$$

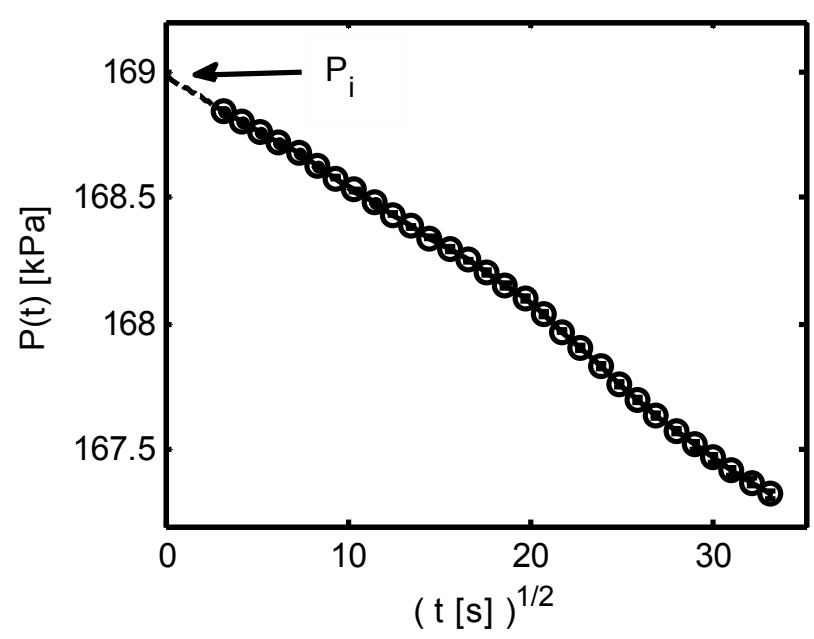

Fig. 12. Typical pressure response curve and definition of $P_{i}$ (absorption of $\mathrm{CO}_{2}$ into isobutanol)

To illustrate the analysis of the pressure response, consider the typical pressure response curve shown in Fig. 12, for the case of absorption of solvent saturated carbon dioxide into a non-aqueous isobutanol layer of $3.5 \pm 0.1 \mathrm{~mm}$ thickness. In Fig. 12, the initial pressure $P_{i}$ immediately after the pressure step is estimated by extrapolating the penetration regime (which is a straight line in a plot of pressure against square-root of time) to $t=0$. In this particular experiment $P_{i}=169.0 \pm 0.6 \mathrm{kPa}$ and initial equilibrium pressure just before the pressure step was $P_{0}=131.8 \pm 0.6 \mathrm{kPa}$. The diffusivity of $\mathrm{CO}_{2}$ in liquid isobutanol is then estimated by least square fitting the normalized pressure $P_{n} \equiv\left(P_{g}(t)-P_{i}\right) /\left(P_{0}-P_{i}\right)$ to Eq. (36). 
Table 1. Operating conditions for tests A, B and C

\begin{tabular}{|c|c|c|c|c|c|c|c|c|c|}
\hline Test & $\begin{array}{c}h \times 10^{3} \\
{[\mathrm{~m}]}\end{array}$ & $\begin{array}{c}P_{i} \times 10^{-3} \\
{[\mathrm{~Pa}]}\end{array}$ & $\begin{array}{c}P_{0} \times 10^{-3} \\
{[\mathrm{~Pa}]}\end{array}$ & $b / h$ & $\begin{array}{c}D_{l} \times 10^{9} \\
{\left[\mathrm{~m}^{2} \mathrm{~s}^{-1}\right]}\end{array}$ & $D_{g} / D_{l}$ & $\begin{array}{c}T \\
{\left[{ }^{\circ} \mathrm{C}\right]}\end{array}$ & $\begin{array}{c}0.03 h^{2} / D_{l} \\
{[\mathrm{~s}]}\end{array}$ & $\begin{array}{c}t_{c} \\
{[s]}\end{array}$ \\
\hline $\mathrm{A}$ & 2.6 & 137.0 & 179.8 & 22.1 & 2.18 & 1994 & 24.5 & 93 & 103 \\
\hline $\mathrm{B}$ & 3.1 & 106.6 & 159.5 & 18.4 & 2.12 & 2279 & 24.0 & 135 \\
\hline $\mathrm{C}$ & 3.5 & 131.8 & 168.6 & 16.1 & 2.07 & 2201 & 22.9 & 176 & 253 \\
\hline
\end{tabular}

The measured diffusivity also provides an additional check for possible defects in the experiment run. In this particular experiment the diffusivity was estimated as $(2.21 \pm 0.04) \times 10^{-9} \mathrm{~m}^{2} \mathrm{~s}^{-1}$ with the system operating at $22.9{ }^{\circ} \mathrm{C}$. This is comparable with the value of $2.20 \times 10^{-9} \mathrm{~m}^{2} \mathrm{~s}^{-1}$ at $25^{\circ} \mathrm{C}$ obtained by Hozawa et. al. (1984). Assuming an instantaneous equilibrium, the true molar flux across the interface is calculated as

$N=-\frac{V}{Z A R T} \frac{d P_{g}}{d t}$

from which the liquid-phase mass transfer coefficient can be determined as

$k_{\text {exp }}=-\frac{N}{C_{b}-C_{i}}$

Since the pressure change in the gas phase is small, we assume that the gas compressibility does not vary appreciably during the course of the experimental runs, and is taken to be a constant value estimated as the compressibility of pure carbon dioxide evaluated at the mean temperature and pressure.

In the situation where the solute has penetrated to the bottom, the penetration theory mass transfer coefficient $k_{p}=\sqrt{D_{l} / \pi t}$ has to be modified to take into account the variation in the bottom boundary concentration $C_{b}$ which is now obtained from Eq. (34). In the finite liquid layer limit, the liquid phase mass transfer coefficient is defined by,

$k_{f}=\frac{-D_{l}\left(\frac{\partial C}{\partial z}\right)_{z=h}}{C_{b}-C_{i}}$

For the finite layer liquid mass transfer coefficient we obtain,

$k_{f}=k_{p} \phi_{l}$

where,

$$
\phi_{l}=\frac{\sum_{n=0}^{\infty}(-1)^{n}\left[\exp \left(-\frac{n^{2} h^{2}}{D_{l} t}\right)-\exp \left(-\frac{(n+1)^{2} h^{2}}{D_{l} t}\right)\right]}{1-\sum_{n=0}^{\infty} 2(-1)^{n} \operatorname{erfc}\left[\left(n+\frac{1}{2}\right)\left(\frac{h^{2}}{D_{l} t}\right)^{\frac{1}{2}}\right]}
$$

The mass transfer enhancement factor is then calculated as

$\Phi=\frac{k_{\exp }}{k_{f}}$

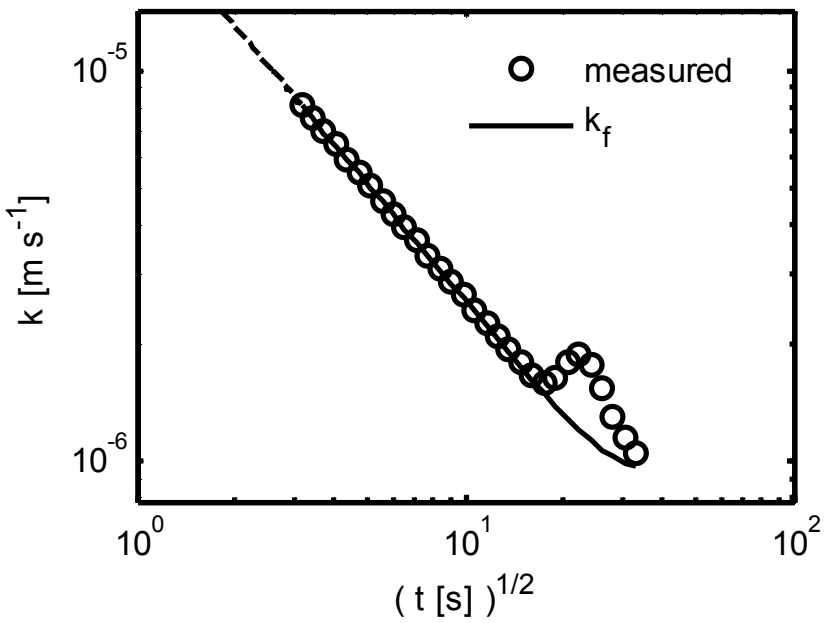

Fig. 13. Variation of mass transfer coefficient with time

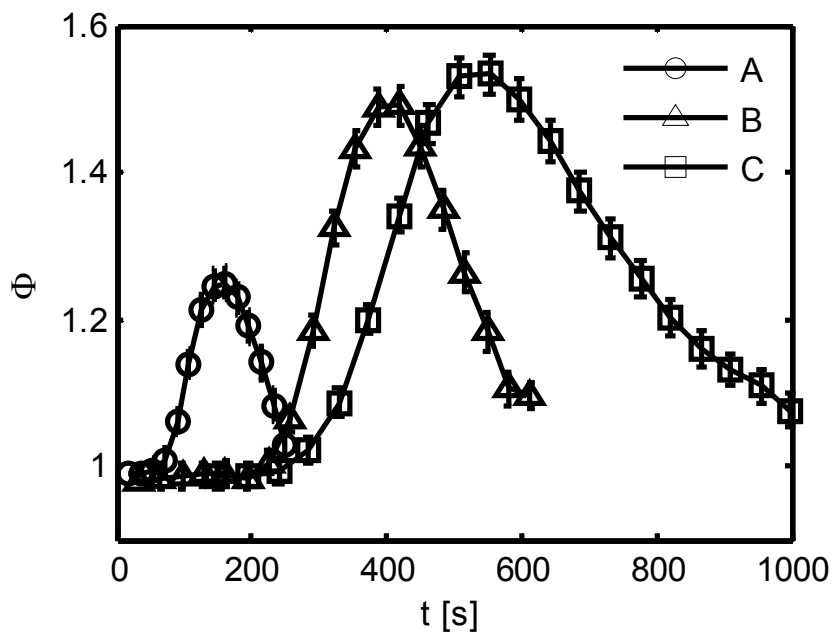

Fig. 14. Collapse of convective mass transfer enhancement

The measured mass transfer coefficients of $\mathrm{CO}_{2}$ into liquid isobutanol are shown in Fig. 13. The circle points in Fig. 13 are the mass transfer coefficients calculated from the measured pressure response using Eq. (40). The measured response clearly shows mass transfer enhancement beginning when $\sqrt{t} \approx 20$ and a collapse of mass transfer enhancement when $\sqrt{t}$ is larger than approximately 25 . The mass transfer enhancement factors measured in three similar experiments are shown in Fig. 14. All three tests are for the physical absorption of pure $\mathrm{CO}_{2}$ into analytical grade liquid isobutanol. The operation conditions for these tests are summarised in Table 1.

\subsection{Comparison with Theoretical Predictions}

In Table 1 the quantity $0.03 h^{2} / D_{l}$ is the dimensioned time corresponding to the marginal point where the solute has just penetrated to the bottom boundary and $t_{c}$ is the experimentally estimated critical 
contact time for the onset of convection. Tests $\mathrm{A}, \mathrm{B}$ and $\mathrm{C}$ reported here have been chosen such that the measured critical contact time is either close to or larger than the marginal point to ensure that the effects of non-diffusing bottom boundary will be significant in these tests. In all three tests a local maximum of mass transfer enhancement factor was observed. The theoretical RBM convection driving force, $\varepsilon=\left(\mathrm{Ra}-\mathrm{Ra}_{c}\right) / \mathrm{Ra}_{c}=\left(\mathrm{Ma}-\mathrm{Ma}_{c}\right) / \mathrm{Ma}_{c}$, under operating conditions of tests $\mathrm{A}, \mathrm{B}$ and $\mathrm{C}$ are shown in Fig. 15.

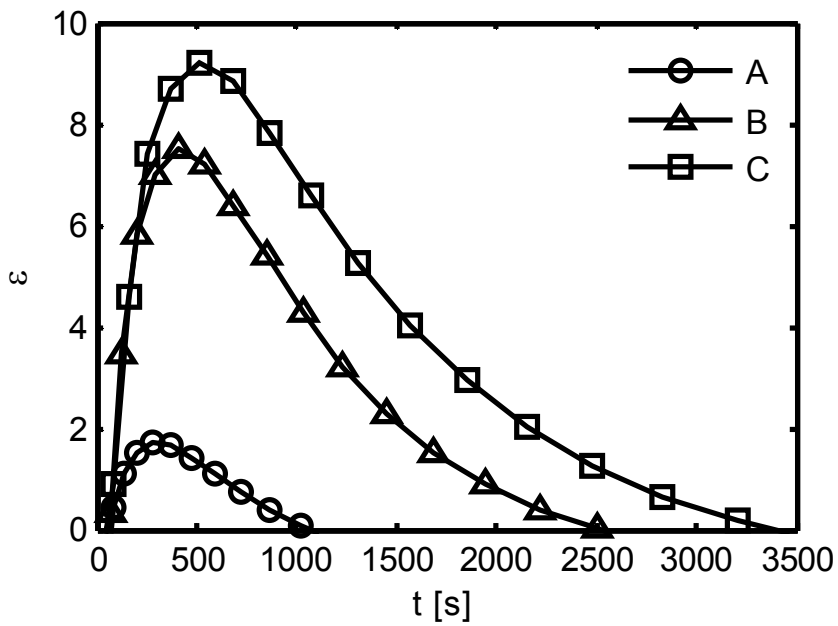

Fig. 15. Theoretical RBM driving force for tests A, B and C

The theoretical critical contact times for test A, B and C were found to be approximately $57 \mathrm{~s}, 58.5 \mathrm{~s}$ and $63.5 \mathrm{~s}$ respectively. The theoretical critical contact times are expected to be lower than measured contact time shown in Fig. 14 since there needs to be a time lag between the critical threshold and the growth of disturbances above the threshold of detection in experiments, and because the effect of surface viscosity has been neglected in the present analysis. However, comparing Fig. 14 and Fig. 15 it is seen that the relative positions of the mass transfer enhancement maxima measured in the experiments (160 $\mathrm{s}, 410 \mathrm{~s}$ and $530 \mathrm{~s}$ for tests $\mathrm{A}, \mathrm{B}$ and $\mathrm{C}$ respectively) are in reasonable agreement with those predicted by the effects of solute transfer to the bottom non-diffusing boundary (301 s, $429 \mathrm{~s}$ and $546 \mathrm{~s}$ for tests A, B and $\mathrm{C}$ respectively). In addition the relative value of the mass transfer enhancement maxima are consistent with the expected RBM driving force, with the measured enhancement maxima increasing from tests $\mathrm{A}$ to $\mathrm{C}$.

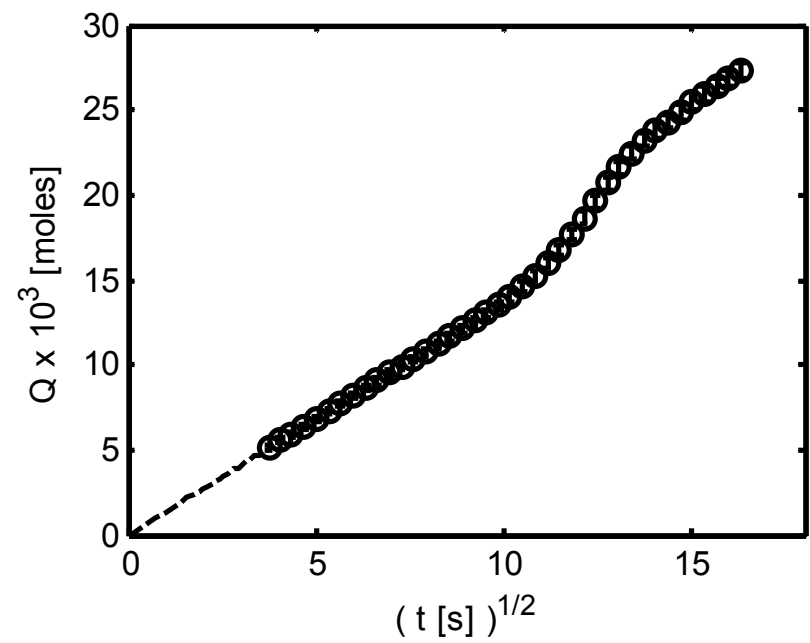

Fig. 16: Collapse of mass transfer enhancement with absorption of $\mathrm{CO}_{2}$ by water.
Figure 16 shows the cumulative moles transferred $Q$ in the absorption of pure carbon dioxide into a water layer approximately 4 $\mathrm{mm}$ thick. The purely diffusive mass transfer close to the initial gasliquid contact time is indicated by the linear relationship between $Q$ and $\sqrt{t}$. The mass transfer rate peaks (maximum slope) at approximately $150 \mathrm{~s}$ from initial contact time which corresponds to a non-dimensionalised contact time $t=0.02$. The carbon dioxide absorption rates measured by Plevan and Quinn (1966) with the water$\mathrm{CO}_{2}$ system do not show such a collapse. This can be expected since the water depth used by Plevan and Quinn was approximately $1 \mathrm{~cm}$ deep, with the reported data terminating at a contact time of approximately 300 s. This corresponds to a non-dimensionalised contact time of $t=0.006$ which is well before $t \approx 0.03$, the approximate time after which the effect of the non-diffusing boundary conditions would become apparent.

\section{CONCLUSIONS}

In a transient gas-liquid solute transfer system, consisting of a thin liquid layer, the stability threshold decreases after initial gas-liquid contact. For short contact times, employing a penetration profile in the stability analysis produces critical Rayleigh and Marangoni in good agreement with that obtained by employing a non-diffusing boundary condition at the bottom interface. During the penetration period both the critical Rayleigh and Marangoni numbers decrease with time, while the system operation Rayleigh and Marangoni numbers remain approximately constant. This behaviour persists for a non-dimensional time greater than approximately 0.03 , provided the gas-liquid thickness ratio is sufficiently large. At longer times when the solute has penetrated to the bottom boundary and penetration theory is no longer valid, a new behaviour is observed in that the critical Rayleigh and Marangoni numbers reach a minimum and thereafter increase. If the time varying concentration at bottom liquid-solid boundary is not severely altered by the onset of convection, then system Rayleigh and Marangoni numbers would also decrease. The onset of convection is then determined by the competition between system operation Rayleigh and Marangoni numbers and critical thresholds. As a result, within certain operation regimes of the system, there can only be a limited period after initial contact when convective instability is possible and within which there is a transient maximum of convective intensity.

Experimental measurement of time varying mass transfer rates in stationary gas-liquid solute transfer supports the theoretical prediction that the Rayleigh-Bénard-Marangoni convection enhanced solute transfer rates should peak and then collapse after longer contact times when penetration theory breaks down and a non-diffusing bottom boundary is in effect. Further experimental verification of the predicted behaviour in this latter regime is required. It is expected that a corresponding flow system would exhibit similar features as the stationary system, which in turn suggests that in a flow system, only a restricted region in the gas-liquid contactor would produce RBM enhanced mass transfer. This opens up the possibility of optimising gasliquid contactors with respect to Rayleigh and Marangoni convection enhanced mass transfer, for instance, by paralleling a limited contact area rather than cascading.

\section{NOMENCLATURE}

$b \quad$ thickness of gas layer (m)

$\mathrm{Bi}$ mass transfer Biot number

C unperturbed concentration of solute in liquid phase $\left(\mathrm{mol} \mathrm{m}^{-3}\right)$

$C^{\prime} \quad$ perturbed concentration $\left(\mathrm{mol} \mathrm{m}^{-3}\right)$

$C_{0} \quad$ liquid phase bulk concentration before gas-liquid contact $\left.(\mathrm{mol} \mathrm{m})^{-3}\right)$

$C_{b} \quad$ concentration at liquid-solid bottom boundary $\left(\mathrm{mol} \mathrm{m}^{-3}\right)$ 
$C_{i} \quad$ interface concentration at $t=0\left(\mathrm{~mol} \mathrm{~m}^{-3}\right)$

$C_{s} \quad$ interface concentration for $t>0\left(\mathrm{~mol} \mathrm{~m}^{-3}\right)$

$D_{g} \quad$ diffusivity of solute in gas phase $\left(\mathrm{m}^{2} \mathrm{~s}^{-1}\right)$

$D_{l} \quad$ diffusivity of solute in liquid phase $\left(\mathrm{m}^{2} \mathrm{~s}^{-1}\right)$

$g \quad$ gravitational acceleration $\left(\mathrm{m} \mathrm{s}^{-2}\right)$

$h \quad$ thickness of liquid layer (m)

$k$

$\mathbf{k}$

wave number $\left(\mathrm{m}^{-1}\right)$ or liquid phase mass transfer coefficient $\left(\mathrm{m} \mathrm{s}^{-1}\right)$

$k_{c g}$

$k_{x}$

$k_{y}$

$\mathrm{Ma}$

$N$

$H$

$P_{g}$

$Q$

$R$

$\mathrm{Ra}$

$t$

$T$

$u$

$U$

$v$

V

$\mathbf{v}^{\prime}$

V

w

$W$

$\hat{U}$

\section{Greek symbols}

$\varepsilon \quad$ RBM driving force

$\alpha_{0} \quad$ solutal expansion coefficient of liquid phase $\left(\mathrm{m}^{3} \mathrm{~mol}^{-1}\right)$

$\phi_{g} \quad$ function defined by Eq. (26)

$\phi_{l} \quad$ function defined by Eq. (43)

$\Phi \quad$ mass transfer enhancement factor

$\mu \quad$ viscosity $\left(\mathrm{N} \mathrm{m}^{-2} \mathrm{~s}\right)$

$v \quad$ kinematic viscosity $\left(\mathrm{m}^{2} \mathrm{~s}^{-1}\right)$

$\rho \quad$ density $\left(\mathrm{kg} \mathrm{m}^{-3}\right)$

$\sigma_{0} \quad$ negative of the slope of the curve of surface tension versus solute concentration $\left(\mathrm{N} \mathrm{m}^{2} \mathrm{~mol}^{-1}\right)$

$\theta \quad$ a small disturbance in concentration $\left(\mathrm{mol} \mathrm{m}^{-3}\right)$

$\Theta \quad$ amplitude of concentration disturbance

\section{Subscripts}

c critical value

exp experimental value

$f \quad$ finite depth

$g \quad$ gas phase

$i \quad$ gas-liquid interface or conditions immediately after a pressures step

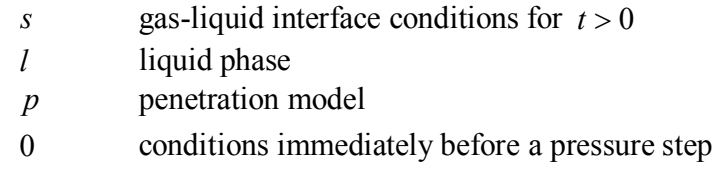

\section{Operators \\ D $\quad \frac{d}{d z}$ \\ $\nabla \quad\left(\frac{\partial}{\partial x}, \frac{\partial}{\partial y}, \frac{\partial}{\partial z}\right)$ \\ $\nabla_{1}^{2} \quad\left(\frac{\partial}{\partial x}, \frac{\partial}{\partial y}\right)$}

$\begin{array}{ll}\text { Abbreviations } \\ \text { BM } & \text { Bénard-Marangoni } \\ \text { PEN } & \text { penetration theory } \\ \text { RB } & \text { Rayleigh-Bénard } \\ \text { RBM } & \text { Rayleigh-Bénard-Marangoni } \\ \text { NDBB } & \text { non diffusing bottom boundary } \\ \text { NDTB } & \text { non-diffusing top boundary }\end{array}$

\section{REFERENCES}

Blair, L. M. and Quinn, J. A., 1969, "Onset of Cellular Convection in a Fluid Layer with Time-Dependent Density Gradients." Journal of Fluid Mechanics 36: 385.

http://dx.doi.org/10.1017/S0022112069001716

Brian, P. L. T., 1971, "Effect of Gibbs Adsorption on Marangoni Instability." Aiche Journal 17(4): 765. http://dx.doi.org/10.1002/aic.690170403

Brian, P. L. T. and Ross, J. R., 1972, "Effect of Gibbs Adsorption on Marangoni Instability in Penetration Mass-Transfer." Aiche Journal 18(3): 582.

http://dx.doi.org/10.1002/aic.690180319

Brian, P. L. T., Vivian, J. E. and Matiatos, D. C., 1967, "Interfacial Turbulence during Absorption of Carbon Dioxide into Monoethanolamine." Aiche Journal 13(1): 28.

http://dx.doi.org/10.1002/aic.690130108

Bird, R. B., Stewart, W. E. and Lightfoot, E. N., 2007, Transport phenomena, J. Wiley

Buffone, C. and Sefiane, K., 2004, "Investigation of thermocapillary convective patterns and their role in the enhancement of evaporation from pores." International Journal of Multiphase Flow 30(9): 1071. http://dx.doi.org/10.1016/j.ijmultiphaseflow.2004.05.010

Byers, C. H. and King, C. J., 1967, "Gas-Liquid Mass Transfer with a Tangentially Moving Interface: Part I. Theory." American Institute of Chemical Engineers Journal 13(4): 628. http://dx.doi.org/10.1002/aic.690130409

Chandrasekhar, S., 1981, Hydrodynamic and hydromagnetic stability, Dover Publications

Choi, C. K., Kang, K. H., Kim, M. C. and Hwang, I. G., 1998, "Convective instabilities and transport properties in horizontal fluid layers." Korean Journal of Chemical Engineering 15(2): 192. http://dx.doi.org/10.1007/BF02707072 
Currie, I. G., 1967, "Effect of Heating Rate on Stability of Stationary Fluids." Journal of Fluid Mechanics 29: 337.

http://dx.doi.org/10.1017/S0022112067000850

Davis, E. J. and Choi, C. K., 1977, "Cellular Convection with LiquidFilm Flow." Journal of Fluid Mechanics 81(3): 565.

http://dx.doi.org/10.1017/S0022112077002237

Debnath, L., 2005, Nonlinear partial differential equations for scientists and engineers, Birkhäuser

Etminan, S. R., Maini, B. B., Chen, Z. X. and Hassanzadeh, H., 2010, "Constant-Pressure Technique for Gas Diffusivity and Solubility Measurements in Heavy Oil and Bitumen." Energy \& Fuels 24: 533. http://dx.doi.org/10.1021/ef9008955

Farajzadeh, R., Barati, A., Delil, H. A., Bruining, J. and Zitha, P. L. J., 2007, "Mass transfer of CO2 into water and surfactant solutions." Petroleum Science and Technology 25(11-12): 1493. http://dx.doi.org/10.1080/10916460701429498

Feit, M. D., Fleck, J. A. and Steiger, A., 1982, "Solution of the Schrodinger-Equation by a Spectral Method." Journal of Computational Physics 47(3): 412. http://dx.doi.org/10.1016/0021-9991(82)90091-2

Gresho, P. M. and Sani, R. L., 1971, "Stability of a Fluid Layer Subjected to a Step Change in Temperature - Transient Vs Frozen Time Analyses." International Journal of Heat and Mass Transfer 14(2): 207.

http://dx.doi.org/10.1016/0017-9310(71)90090-1

Hassanzadeh, H., Pooladi-Darvish, M. and Keith, D. W., 2009, "The Effect of Natural Flow of Aquifers and Associated Dispersion on the Onset of Buoyancy-Driven Convection in a Saturated Porous Medium." Aiche Journal 55(2): 475.

http://dx.doi.org/10.1002/aic.11664

Hozawa, M., Komatsu, N., Imaishi, N. and Fujinawa, K., 1984, "Interfacial Turbulence During The Physical Absorption of Carbon Dioxide into Non-Aqueous Solvents." Journal of Chemical Engineering of Japan 17(2): 173.

http://dx.doi.org/10.1252/jcej.17.173

Kim, M. C., Choi, C. K., Yoon, D. Y. and Chung, T. J., 2007, "Onset of marangoni convection in a horizontal fluid layer experiencing evaporative cooling." Industrial \& Engineering Chemistry Research 46(17): 5775.

http://dx.doi.org/10.1021/ie070006y

Lebon, G. and Cloot, A., 1982, "Buoyancy and Surface-Tension Driven Instabilities in Presence of Negative Rayleigh and Marangoni Numbers." Acta Mechanica 43(3-4): 141.

http://dx.doi.org/10.1007/BF01176278

Lick, W. J., 1964, "The Propagation of Small Disturbances in a Radiating Gas." Journal of Fluid Mechanics 18(2): 274.

http://dx.doi.org/10.1017/S0022112064000192

Lu, H. H., Yang, Y. M. and Maa, J. R., 1997, "Surfactant effects on absorption in the presence of induced interfacial turbulence." Aiche Journal 43(7): 1909.

http://dx.doi.org/10.1002/aic.690430726

Nield, D. A., 1964, "Surface Tension and Buoyancy Effects in Cellular Convection." Journal of Fluid Mechanics 19: 341.

http://dx.doi.org/10.1017/S0022112064000763
Palmer, H. J. and Berg, J. C., 1971, "Convection Instabilitis in Liquid Pools Heated from Below." Journal of Fluid Mechanics 47: 779. http://dx.doi.org/10.1017/S0022112071001368

Pearson, J. R., 1958, "On Convective Cells induced by Surface Tension." Journal of Fluid Mechanics 4: 489. http://dx.doi.org/10.1017/S0022112058000616

Plevan, R. E. and Quinn, J. A., 1966, "Effect of Monomolecular Films on Rate of Gas Absorption into a Quiescent Liquid." AIChE Journal 12(5): 894.

http://dx.doi.org/10.1002/aic.690120512

Rayleigh, L., 1916, "On Convection Currents in a Horizontal Layer of Fluid, when the Higher Temperature is on the Underside." Philosophical Magazine 32(192).

Scheid, B., Kalliadasis, S., Ruyer-Quil, C. and Colinet, P., 2008, "Interaction of three-dimensional hydrodynamic thermocapillary instabilities in film flows." Physical Review E, Statistical, Nonolinear, Soft Matter 78: 066311.

http://dx.doi.org/10.1103/PhysRevE.78.066311

Scriven, L. E. and Sternling, C. V., 1964, "On Cellular Convection Driven by Surface-Tension Gradients - Effects of Mean Surface Tension and Surface Viscosity." Journal of Fluid Mechanics 19(3): 321.

http://dx.doi.org/10.1017/S0022112064000751

Sefiane, K. and Cameron, J., 2006, "Modelling of heat and fluid flow during the evaporation of volatile drops on hot substrates." Progress in Computational Fluid Dynamics 6(6): 363. http://dx.doi.org/10.1504/PCFD.2006.010778

Sheikha, H., Pooladi-Darvish, M. and Mehrotra, A. K., 2005, "Development of graphical methods for estimating the diffusivity coefficient of gases in bitumen from pressure-decay data." Energy \& Fuels 19(5): 2041.

http://dx.doi.org/10.1021/ef050057c

Sparrow, E. M., Goldstein, R. J. and Jonsson, V. K., 1964, "Thermal Instability in a Horizontal Fluid Layer - Effect of Boundary Conditions and Non-Linear Temperature Profile." Journal of Fluid Mechanics 18(4): 513.

http://dx.doi.org/10.1017/S0022112064000386

Sun, Z., 2006, "Onset of Rayleigh- Bénard- Marangoni Convection in Gas- Liquid Mass Transfer with Two-Phase Flow: Comparison of Measured Results with Theoretical Results." Ind. Eng. Chem. Res 45(18): 6325.

http://dx.doi.org/10.1021/ie060178f

Sun, Z. F. and Fahmy, M., 2006, "Onset of Rayleigh-BenardMarangoni convection in gas-liquid mass transfer with two-phase flow: Theory." Industrial \& Engineering Chemistry Research 45(9): 3293. http://dx.doi.org/10.1021/ie051185r

Sun, Z. F. and Yu, K. T., 2006, "Rayleigh-Benard-Marangoni cellular convection - Expressions for heat and mass transfer rates." Chemical Engineering Research \& Design 84(A3): 185.

http://dx.doi.org/10.1205/cherd.05057

Tharanivasan, A. K., Yang, C. D. and Gu, Y. G., 2004, "Comparison of three different interface mass transfer models used in the experimental measurement of solvent diffusivity in heavy oil." Journal of Petroleum Science and Engineering 44(3-4): 269.

http://dx.doi.org/10.1016/j.petrol.2004.03.003 
Trefethen, L., 2000, Spectral methods in MATLAB, Society for Industrial and Applied Mathematics

Vidal, A. and Acrivos, A., 1966, "Nature of the Neutral State in Surface-Tension Driven Convection." Physics of Fluids 9: 615. http://dx.doi.org/10.1063/1.1761716

Weideman, J. A. C. and Reddy, S. C., 2000, "A MATLAB differentiation matrix suite." Acm Transactions on Mathematical Software 26(4): 465.

http://dx.doi.org/10.1145/365723.365727

Zhang, Y. P., Hyndman, C. L. and Maini, B. B., 2000, "Measurement of gas diffusivity in heavy oils." Journal of Petroleum Science and Engineering 25(1-2): 37.

http://dx.doi.org/10.1016/S0920-4105(99)00031-5

\section{APPENDIX A: GAS PHASE MASS TRANSFER COEFFICIENT}

To obtain the concentration profile of solute in the gas phase, consider a vertical coordinate axis $z_{g}$ with the origin placed at the top gas-solid boundary of the system shown in Fig. 1, and the positive $z_{g}$ direction pointing vertically downward. Then, by analogy with Eqs. (1) to (5), the solute concentration profile in the gas-phase is given by,

$$
\begin{aligned}
& \frac{C_{g}\left(t, z_{g}\right)-C_{g, 0}}{C_{g, i}-C_{g, 0}} \\
& \quad=\sum_{n=0}^{\infty}(-1)^{n}\left[\operatorname{erfc}\left(\frac{(2 n+1) b-z_{g}}{\sqrt{4 D_{g} t}}\right)+\operatorname{erfc}\left(\frac{(2 n+1) b+z_{g}}{\sqrt{4 D_{g} t}}\right)\right]
\end{aligned}
$$

where $D_{g}$ is the diffusivity of solute in the gas phase consisting of solute and the vapour of the liquid phase species, $b$ is the gas phase thickness, $C_{g, 0}$ is the initial uniform solute concentration in the gas phase and $C_{g, i}$ is solute concentration at the gas-liquid interface $z_{g}=b$. The solute concentration at the gas-solid boundary
$C_{g, b}=C_{g}(t, b)$, is then given by,

$C_{g, b}=C_{g, 0}-\left(C_{g, 0}-C_{g, i}\right) \sum_{n=0}^{\infty}(-1)^{n}\left[2 \operatorname{erfc}\left(\frac{(2 n+1) b}{\sqrt{4 D_{g} t}}\right)\right]$

and the solute flux $N=-D_{g}\left(\partial C_{g} / \partial z_{g}\right)_{z_{g}=b}$ across the gas-liquid interface is given by,

$N=D_{g}\left(C_{g, 0}-C_{g, i}\right) \sum_{n=0}^{\infty}(-1)^{n}\left\{\frac{\exp \left[-\frac{n^{2} b^{2}}{D_{g} t}\right]-\exp \left[-\frac{(n+1)^{2} b^{2}}{D_{g} t}\right]}{\sqrt{\pi D_{g} t}}\right\}$

The gas-phase mass transfer coefficient is defined by (Bird, et al., 2007),

$k_{c g}=\frac{N}{C_{g, b}-C_{g, i}}$

Substituting Eqs. (46) and (47) into Eq. (48), we obtain,

$$
k_{c g}=\left(\frac{D_{g}}{\pi t}\right)^{\frac{1}{2}} \frac{\sum_{n=0}^{\infty}(-1)^{n}\left\{\exp \left[-\frac{n^{2} b^{2}}{D_{g} t}\right]-\exp \left[-\frac{(n+1)^{2} b^{2}}{D_{g} t}\right]\right\}}{1-\sum_{n=0}^{\infty} 2(-1)^{n} \operatorname{erfc}\left[\frac{(2 n+1) b}{\left.\sqrt{4 D_{g} t}\right]}\right.}
$$

Since the solute flux at the interface determined from the gas side must balance the solute flux as determined from the liquid side, it is convenient to introduce the non-dimensionalising distance scale $h$ and time scale $h^{2} / D_{l}$. This can be achieved by defining the parameter $Z_{n}=\left(D_{l} / D_{g}\right)(b / h)^{2}\left(h^{2} / D_{l} t\right)$ as given in Eq. (27). Then, the second factor in Eq. (49), immediately becomes the term $\phi_{g}$ as given by Eq. (26). 